\title{
Allaitement - une autre scène d'interventions nuisibles
}

\section{Jean Martin}

Dr méd., membre de la rédaction

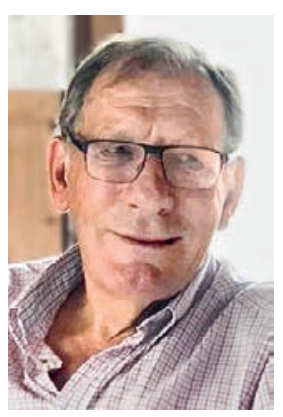

Chaque année, Genève voit se dérouler l'Assemblée mondiale de la santé (AMS). Elle a pour but de suivre l'activité de l'OMS et de discuter ses orientations stratégiques, son budget, etc. Elle a ainsi, évidemment, des dimensions de santé publique mais est aussi très diplomatique - avec des manœuvres qui n'ont pas pour but premier la meilleure santé de la planète. Un exemple marquant en a été donné en mai dernier.

De longue date, l'OMS promeut activement l'allaitement au sein. On se souvient des batailles des années 1970, où Nestlé avait été traité de «baby killer». J'ai alors fait partie d'une délégation reçue au siège de la firme, à Vevey. Les dirigeants rencontrés protestaient vivement de l'excellence de leurs produits et de leurs intentions mais ils avaient beaucoup de peine à comprendre les effets délétères, dans les régions pauvres en particulier, de la publicité pour les substituts du lait maternel: pratiquement, les mères donnaient alors le plus sou-

\section{Des intérêts de pur lucre cherchent trop souvent à affaiblir des recommandations majeures.}

vent à leurs enfants un breuvage dilué (jugeant que si le liquide du biberon était blanc, il était adéquat...). De plus, l'eau utilisée était contaminée. D’où le funeste cercle vicieux «infection menant à la malnutrition, qui rend le nourrisson plus sensible à l'infection» - abondamment démontré par les études faites. Avec souvent une issue fatale. Il était donc complètement justifié de demander l'interdiction de toute incitation à utiliser les substituts du lait maternel - et de toutes «offres spéciales» ou cadeaux aux mères ou aux professionnels de santé! D'où l'adoption en 1981 du Code international de commercialisation de ces substituts. Il est utile de rappeler que la pratique de l'allaitement au sein est déterminée beaucoup plus par des facteurs culturels que biologiques: dans les sociétés où il est attendu que la mère allaite, la très grande majorité d'entre elles le fait (l'impossibilité physique/biologique est rarissime). Ainsi dans beaucoup de sociétés rurales. Dans ces groupes très "allaitants", alléguer que beaucoup de femmes auraient de la peine à donner le sein est destructeur, d'où l'importance de lutter contre tout tel message.
Près de quarante ans après le Code de 1981, l'AMS 2018 a vu un retour caricatural du lobbying s'efforçant de pousser la vente de substituts, au détriment de la santé des enfants (même si ces produits sont "techniquement» corrects). Le magazine Time du 23 juillet 2018 décrit, reprenant un article du New York Times du 8 juillet [1], la démarche mercantile des Etats-Unis à cette Assemblée: l'Administration Trump a voulu diluer une résolution visant à renouveler l'engagement à promouvoir l'allaitement au sein et, plus généralement, à restreindre la promotion d'aliments susceptibles de conséquences nuisibles pour les enfants (sucre en excès, etc.). Sa démarche ayant échoué, Washington a passé à la politique du "gros bras»: ses représentants se sont tournés vers l'Equateur, initiateur de la résolution, de manière tout à fait sèche: si vous ne renoncez pas, les Etats-Unis prendront des mesures punitives dans le domaine commercial et de l'aide militaire. L'Equateur s'est soumis... à la consternation de diplomates et experts présents. Interpellée, l'Administration US a indiqué en toute simplicité qu'elle l'avait fait pour soutenir les firmes produisant des substituts. Les médias sont très critiques de ce chantage. Remarque de Time toutefois: «Qui a l'autorité de dire quand renoncer à un bénéfice [ici, celui pour l'enfant d'être allaité] devient dangereux? Ces décisions sont rendues d'autant plus brouillées par un 'marketing digital hyper-concentré'; ainsi sur plusieurs débats chauds à propos de vaccination par exemple, ou de traitement du déficit d'attention - ADHD. Les mères doivent s'efforcer de filtrer, dans le flou et l'exagéré, ce qui est correct/ raisonnable, tout en sachant qu'il y aura toujours quelqu'un pour dire qu'elles ont tort.» Et citant la présidente de l'American Academy of Pediatrics disant «la décision d'allaiter au sein n'est pas un choix de style de vie, c'est une décision d'importance critique pour le petit enfant».

Cette récente tentative de faire passer le profit avant la santé - et la santé des plus faibles - souligne l'importance de maintenir un regard critique (objectif mais ferme) et de corriger les fake news - pas facile dans une société où l'information discutable fleurit sur les réseaux. Des intérêts de pur lucre cherchent trop souvent à affaiblir des recommandations majeures. 